\title{
Parallel P-PD controller to achieve vibration and position control of a flexible beam
}

\author{
Ammar N. Abbas, Muhammad Asad Irshad \\ Department of Mechatronics Engineering, \\ École Nationale Supérieure De Mécanique Et Des Microtechniques (ENSMM), France
}

\begin{tabular}{l} 
Article Info \\
\hline Article history: \\
Received Sep 19, 2020 \\
Revised Mar 6, 2021 \\
Accepted Apr 9, 2021 \\
\hline
\end{tabular}

Keywords:

Flexible beam Industrial robotics

Parallel control

Vibration control

\begin{abstract}
Robotic arms are considered as a cantilever beam fixed at one end and due to the length-to-weight ratio, it has a significant vibration-induced that needs to be controlled to achieve accurate position, speed control and to increase its efficiency. In this project, a discretized Timoshenko beam model is used to discuss the dynamics of the system. Further, to implement the control on the hardware an experimental setup is fabricated to observe the open-loop and closed-loop responses of the beam made of low-density polyethylene. An accelerometer as a feedback sensor is attached at one end of the flexible beam while another end is fixed at the moving cart having DC motor as an actuator. Simulink is used as the programming tool to perform all of the experimentation. Proportional-integral-derivative (PID) tuning is performed. Following that open-loop responses of the deflection of the beam parallel to the motion are observed with different input waveforms. By applying a proportional control scheme, another experiment is performed to demonstrate the disturbance rejection with an accelerometer as a feedback sensor, while ignoring position control. Finally, a PD and P based parallel control scheme is proposed to obtain simultaneously both position control and vibration reduction.
\end{abstract}

This is an open access article under the CC BY-SA license.

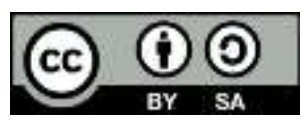

Corresponding Author:

Ammar N. Abbas

Department of Mechatronics Engineering

E NSMM - École Nationale Supérieure De Mécanique Et Des Microtechniques

26 Rue de l'Épitaphe, 25000, Besancon, France

Email: ammar.abbas@eu4m.eu

\section{INTRODUCTION}

This project consists of making a Cartesian robot composed of a flexible beam being moved on guiding rails actuated by a DC motor in order to control the vibration, which is considered one of the major problems of engineering systems [1]. It is necessary to control this vibration in order to reduce energy loss and make the system efficient [2]. The work is divided into different parts such as design, assembly, and development of the control law, for which Ziegler Nichols tuning method of proportional-integral-derivative (PID is used [3]. The control law must be able to reduce the vibrations of the beam during the motion. The manipulation would possibly consist of a motor, an angular-linear transformation belt, a carriage, and a flexible beam. The deformation of the beam would be captured by an accelerometer while the position is measured with an encoder. Some application of this system includes an automated storage and retrieval systems, robotic links, satellites, airplane wings, and many other systems where cantilever beam is being used.

An observer-based control strategy was proposed by Dadfarnia et al. [4] for a flexible cantilever beam of length $300 \mathrm{~mm}$, having translatory base support. PD control is applied for translation control while Lyapunov based controller was designed for controlling the piezoelectric actuator. Hu et al. [5] proposed a 
linear quadratic state-feedback regulator and mode theory to minimize vibration of flexible link with piezoelectric actuator and strain gauge transducer. The experimented result emphasized good disturbance rejection and rapid strain reduction. Qiu et al. [6] proposed an accelerometer-based active vibration control for a cantilever beam. A piezoelectric actuator is used for experimental analysis. Non-collocated sensor and piezoelectric actuator dynamical model was derived for designing proportional feedback control of acceleration. Experimental results demonstrated the effectiveness of the proposed control scheme [6]. Song and $\mathrm{Gu}$ [7] presented the active vibration control performance of a flexible beam by using piezoceramic sensors and actuators. They adopted a sliding mode controller which has inherent robustness to system parameter variation and external disturbances.

While modelling this kind of complex system, there is always some engineering approximation that comes into consideration. At some points, we ignore dynamics and high-frequency modes (as they have a limited role in determining essential model characteristics than low-frequency modes) to get a reduced system that might result in reduced stability and uncertainties. All such approximation techniques have some pros and cons that enabled us to work with a complex system in constrained space.

In this project, a PD and P based parallel control scheme is proposed to obtain simultaneously both the position control and the vibration reduction. The composition of this report is as follows: Section 2 describes project methodology 3-D modelling, Beam analysis, sensor, actuator, and controller. Section 3 demonstrates a brief description of the experimental setup, and development of the control law. Section 4 discusses the results. Finally, the conclusion is derived from experimentation with discussion on prospects.

\section{METHODOLOGY}

This section includes the proposed methodology used to control and actuate the system. The dynamic analysis of a flexible beam is briefly discussed along with the finite element analysis. The block diagram representation is discussed to illustrate the working environment for the programming and control.

\subsection{Beam model analysis}

For the dynamic vibration analysis of the beam, Timoshenko beam model representation is used with discretization parameters [8].

$$
\begin{aligned}
& p(z) \frac{\partial^{2} w}{\partial t^{2}}(z, t)=\frac{\partial}{\partial z}\left[K(z)\left(\frac{\partial w}{\partial z}(z, t)-\emptyset(z, t)\right)\right], z \in(a, b), t \geq 0 \\
& I_{p}(z) \frac{\partial^{2} \emptyset}{\partial t^{2}}(z, t)=\frac{\partial}{\partial_{z}}\left(E I(z) \frac{\partial \emptyset}{\partial z}(z, t)\right)+K(z)\left(\frac{\partial w}{\partial z}(z, t)-\emptyset(z, t)\right)
\end{aligned}
$$

\subsubsection{Beam parameters}

A low-density polyethylene material beam of $300 \mathrm{~mm}$ length, a width of $35 \mathrm{~mm}$, and thickness of $2.5 \mathrm{~mm}$ is used in this experiment with material properties of density: $0.92 \mathrm{~g} / \mathrm{cm} 3$, Young's modulus: $0.1 \mathrm{e}-0.3$ GPa, and bulk's modulus: 1.7 GPa. This beam is fixed on one end with the cart that reduces it is workable length by $40 \mathrm{~mm}$. Beam specifications are shown in Table 1 .

Table 1. Beam specifications

\begin{tabular}{cc}
\hline Material & low-density Polyethylene \\
\hline Dimensions & $300 * 35^{*} 2.5 \mathrm{~mm}$ \\
Density & $0.92 \mathrm{~g} / \mathrm{cm} 3$ \\
Young's modulus & $0.1 \mathrm{e}-0.3 \mathrm{GPa}$ \\
Bulk's modulus & $1.7 \mathrm{GPa}$ \\
\hline
\end{tabular}

\subsubsection{ANSYS simulation for critical analysis}

We simulated different types of beams to observe if they met our project specifications. Due to translation, a low force is going to be applied to the beam. Given sensor limitations, we need reasonable deflections/vibration that can be detected and transmitted for analysis. Firstly, we used an aluminium beam of length $300 \mathrm{~mm}$, width $20 \mathrm{~mm}$, and thickness of $1.2 \mathrm{~mm}$. Fixing one side with support and on the other side, applying $0.075 \mathrm{~N}$ force in negative Y-direction produced $2.12 \mathrm{~mm}$ deformation as shown in Figure 1.

Then reconsidering options, we opted for a low-density polyethylene material-based plastic beam of length $300 \mathrm{~mm}$, the width of $35 \mathrm{~mm}$, and thickness of $3.5 \mathrm{~mm}$. Applying the same force resulted in better deformations around $25 \mathrm{~mm}$. We selected this beam for further analysis as our anticipation for the actual model was to get 20-30 mm deflection in response to applied force as shown in Figure 2. 


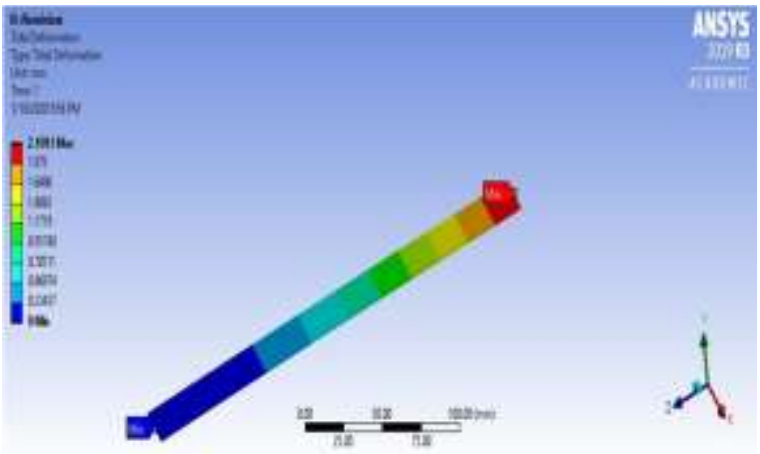

Figure 1. ANSYS aluminium with an applied force of $0.075 \mathrm{~N}$ in negative Y-direction

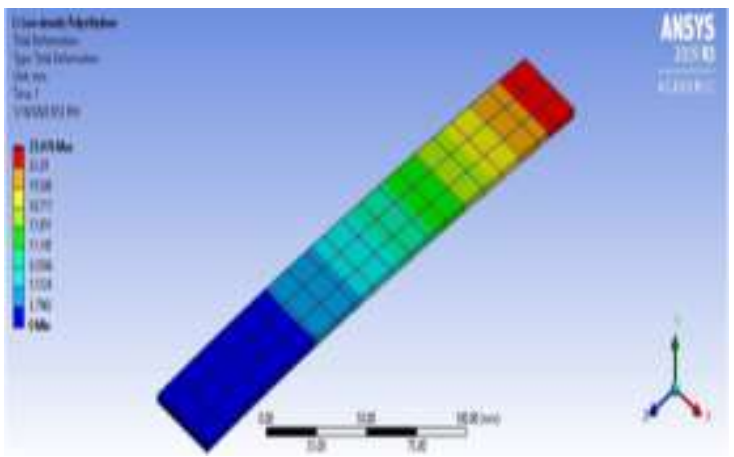

Figure 2. A low-density Polyethylene with an applied force of $0.075 \mathrm{~N}$ in negative $\mathrm{Y}$-direction

\subsection{D Model}

To make a base structure for the cart, a 3D model was created as shown in Figure 3. An experimental prototype was fabricated through laser cutting technology on plexiglass material. To move the cart back and forth two translatory guides are passed through the cart. To minimize friction between the cart and the guides 4 linear bearings are added at each side.

\subsection{Schematic and block diagram}

The motion is provided by a DC motor connected through a belt-pulley drive. The belt is fixed at both ends of the cart to provide direct motion and runs through a free pulley at the other end. The beam is attached to the cart using a nut and bolt. The system schematic is shown in Figure 4 proposes the subsystems:

\subsubsection{Sensors}

Two sensors are used in this setup. To achieve position feedback, control a hall sensor-based encoder is attached to the motor and used to measure the position in terms of pulse-interrupts through the sensor that measures 29 pulses/cm. To measure the deflection/ vibration of the beam an accelerometer is attached at the tip of the beam, where maximum deformation is observed as shown with the finite element analysis tool in the previous section. The accelerometer is configured for 8-bit resolution with 64 counts/g and a maximum range of $2 \mathrm{~g}(\mathrm{~g} \approx 9.81 \mathrm{~m} / \mathrm{s} 2)$ to maintain the trade-off between the resolution, sampling rate, and noise of measurement [9]. The $\mathrm{z}$-axis of the accelerometer is positioned to be along with the motion of the cart. The $\mathrm{x}$-axis is towards the fixed end of the beam and the $y$-axis towards the base. The deflection sensor can overcome the error of non-collocated sensor modelling that is the limitation of using an accelerometer [10-15].

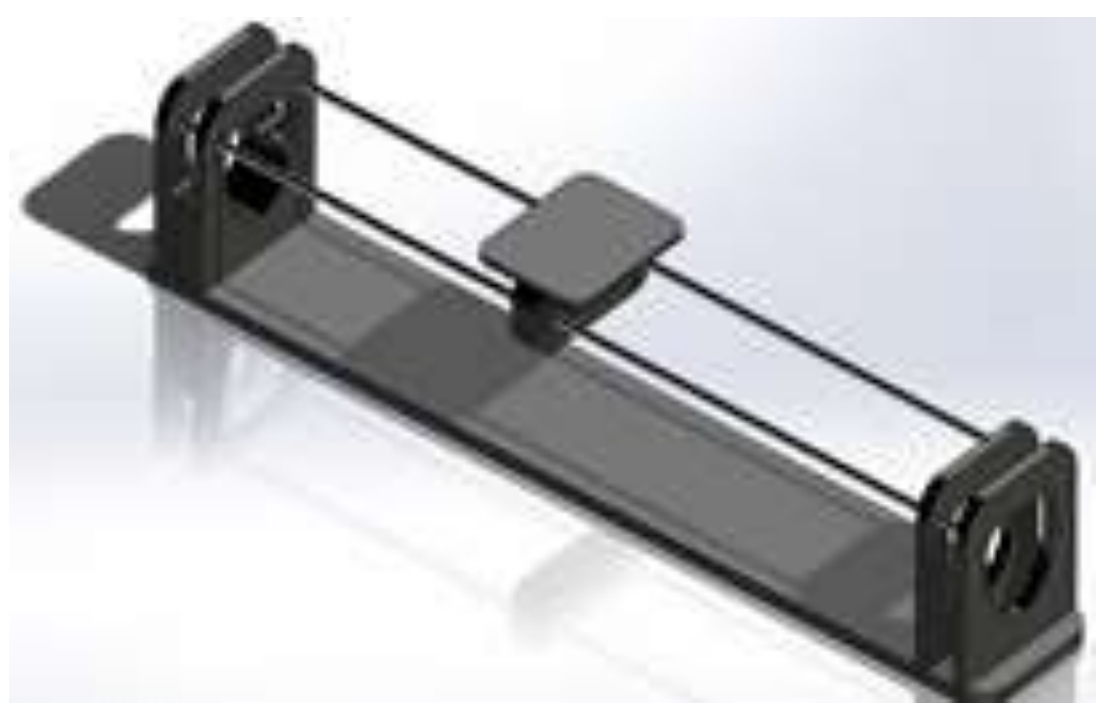

Figure 3. 3D CAD model of the base structure 

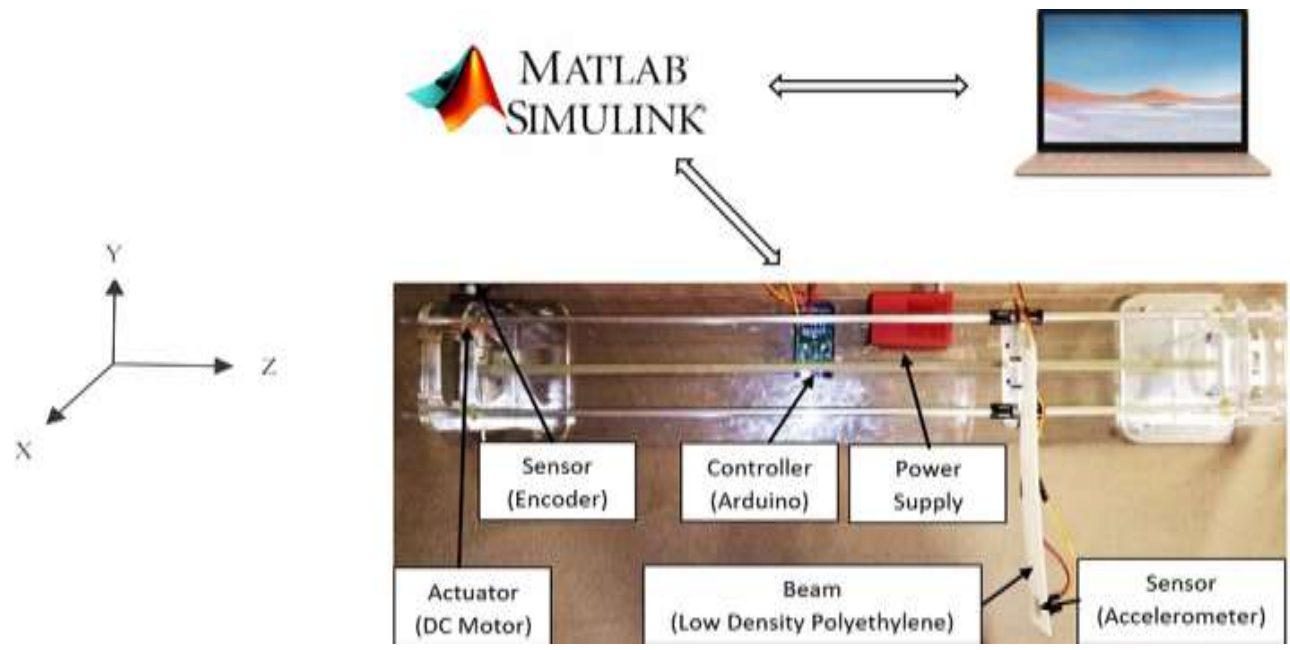

\subsubsection{Actuator}

Figure 4. Block schematic for the experimental setup

DC motor with an encoder is used as an actuator to provide the motion to the system (cart + beam). The motor specifications are given in the Table 2.

\begin{tabular}{cc} 
Table 2. Actuator specifications \\
\hline Operating voltage & $12 \mathrm{~V} \mathrm{D.C.}$ \\
\hline Related torque & $0.3 \mathrm{kgf.cm}$ \\
No-load current & $200 \mathrm{~mA} \mathrm{max}$. \\
No-load speed & $789 \mathrm{rpm}$ \\
Stall current & $2.4 \mathrm{~A}$ \\
Stall torque & $2.2 \mathrm{kgf.cm}$ \\
Reduction ratio & $1 / 19.225$ \\
\hline
\end{tabular}

\subsubsection{Controller}

Arduino Mega 2560 is used as the microcontroller to control the system. The communication protocol between Accelerometer and controller is $I^{2} C$ based, while the encoder is communicating through the UART protocol. To program the board, Simulink is used as the programming tool to create a communication channel between the controllers. An external mode-based environment execution allows the users to deploy the block diagram generated codes onto the controller. This feature is useful to observe as well as control the parameters in real-time.

\section{EXPERIMENTAL ANALYSIS}

In this section, the control methodology is illustrated through Simulink block diagrams. Several control algorithms were adopted to show the different trends of deflection at tip, reference tracking, and control input.

\subsection{Position control}

The Simulink block diagram shown in Figure 5 is used for reference tracking using PD Controller tuned using the Ziegler Nichols tuning method. The subsystem block of the motor consisting of speed, direction, and switch blocks, is used to drive the motor, that is connected to the controller and power supply by a motor driver. A comparator block is used to determine the direction of the motor from the control signal for the positive or negative control input. A saturation block is used to marginalize the pulse width modulation (PWM) input to the motor speed pin with a range of dead zone (10) and a maximum of 255 . These boundary conditions refer to the minimum voltage required by the motor to overcome the static friction and the maximum PWM bandwidth allowed on the controller pin. Encoder block reads the pulses using a hall effect sensor that sends an interrupt signal at every pulse. This output (in pulses) is used to compute the error between the desired and measured positions. By using a multiplying factor, one can directly use the position as input instead of pulses. 


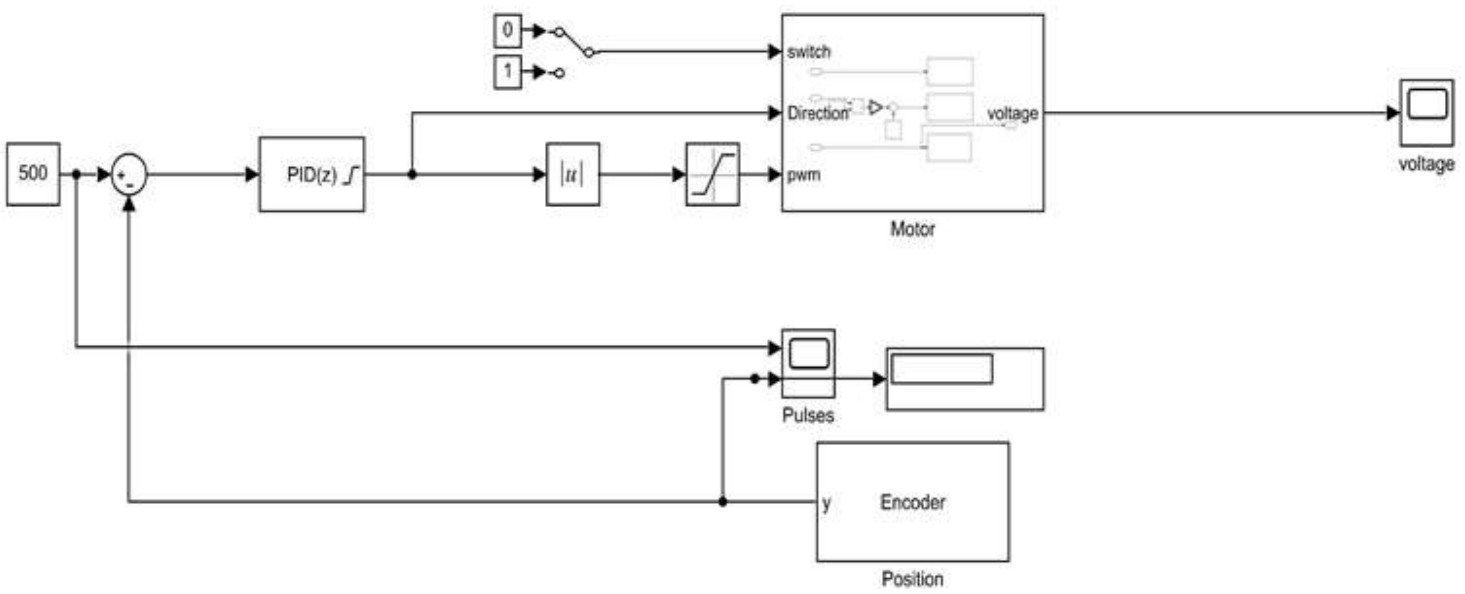

Figure 5. Simulink block diagram for position feedback control

\subsubsection{Different input signals with $x, y$, and $\mathrm{z}$ deflection data}

To observe the beam deflection trends in the $\mathrm{x}, \mathrm{y}$, and $\mathrm{z}$-axis, different input signals (sine, square, and triangle) were used in the position feedback control loop as shown in Figure 6. The scope is used to observe the reference tracking. Beam deflection in terms of acceleration in $\mathrm{x}, \mathrm{y}$, and $\mathrm{z}$ is plotted in a single scope. To make a fair comparison that indicates a maximum change in each axis, the gravitational effect was removed by adding a dead zone block to the output along the y-axis (downwards). Results are shown in section 4 with a brief description.
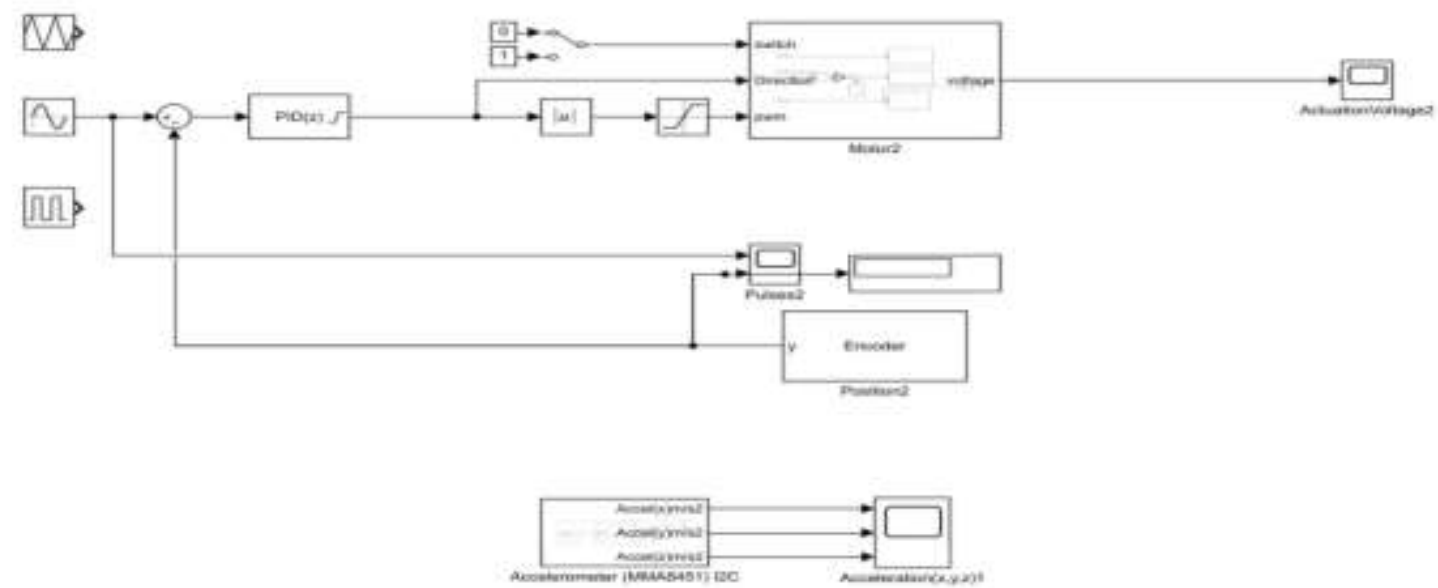

Figure 6. Simulink block diagram for different input signals and open-loop responses for beam deflection $(\mathrm{x}, \mathrm{y}, \mathrm{z})$

\subsection{Vibration control}

To control the vibration/ deflection of the beam along the axis of motion, the Simulink control block diagram was developed as shown in Figure 7. The measured beam deflection in terms of acceleration is used to compute the error signal in a P controller-based feedback loop. 


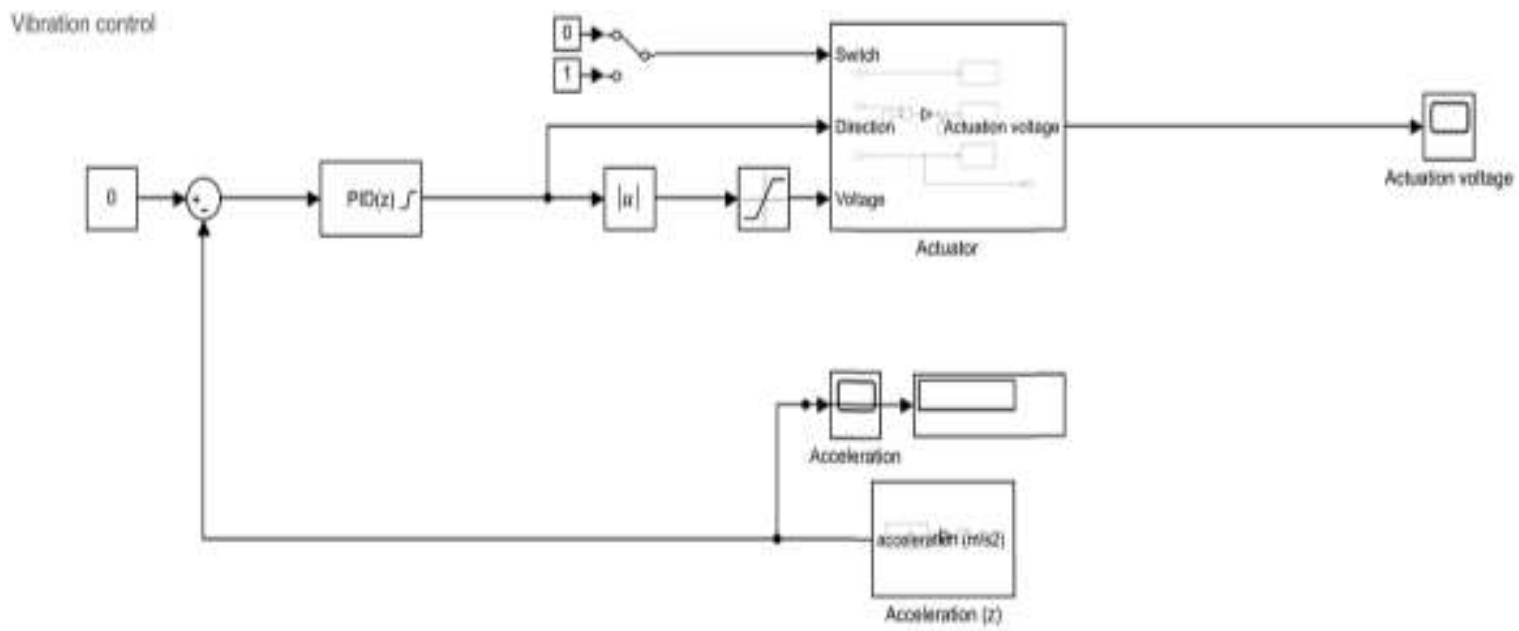

Figure 7. Simulink block diagram for vibration (acceleration(z)) feedback control

\subsection{Parallel control}

To achieve position and vibration control a parallel feedback loop is proposed to integrate both in a single configuration as shown in Figure 8. Different control inputs can be configured to the system to achieve reference tracking and disturbance rejection. The PD-gain computed from the position feedback is added to the P-gain computed from the vibration feedback. This methodology proved its effectiveness as shown by results in the section.

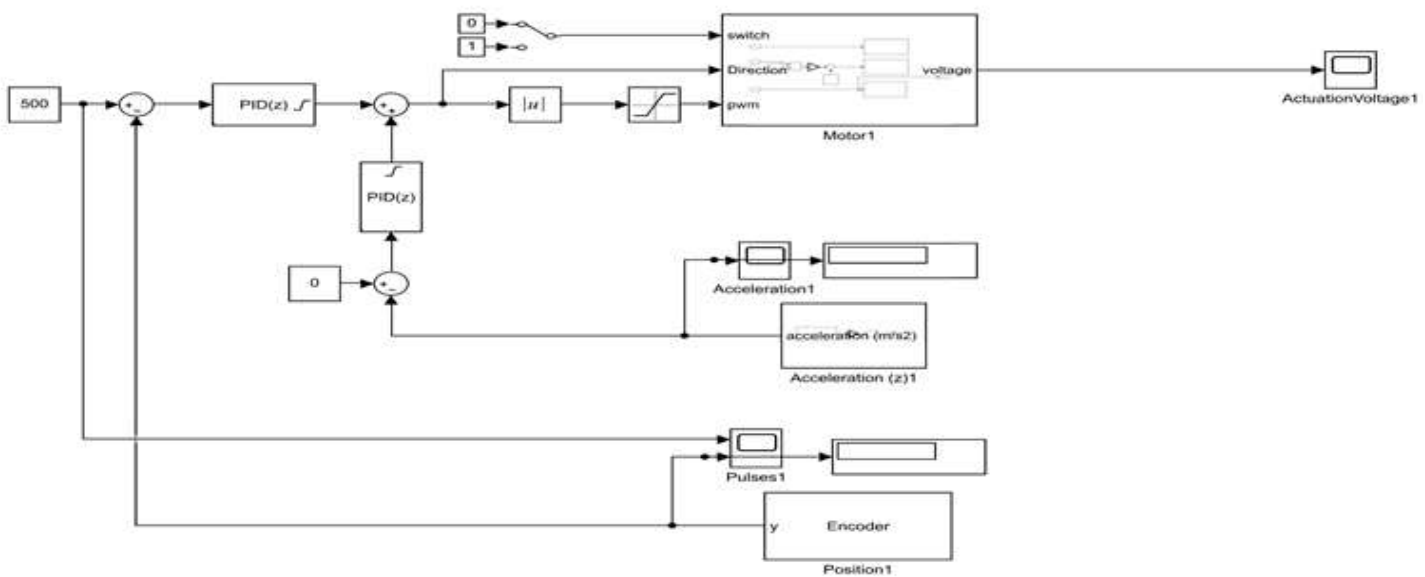

Figure 8. Simulink block diagram for parallel control with position and vibration feedback gains

\subsubsection{Disturbance rejection}

Disturbance rejection was observed on the parallel control with set position tracking in two ways, one applying the impulse force through Simulink to the system for $0.5 \mathrm{~s}$ of an amplitude of 200 as shown in Figure 9. This applies the disturbance to the cart which translates the vibration in the beam passing through the fixed end. In the second method, a force is applied directly to the beam externally causing it to vibrate along the z-axis, the actuator compensates for this disturbance and maintains the position of the cart in both cases. 


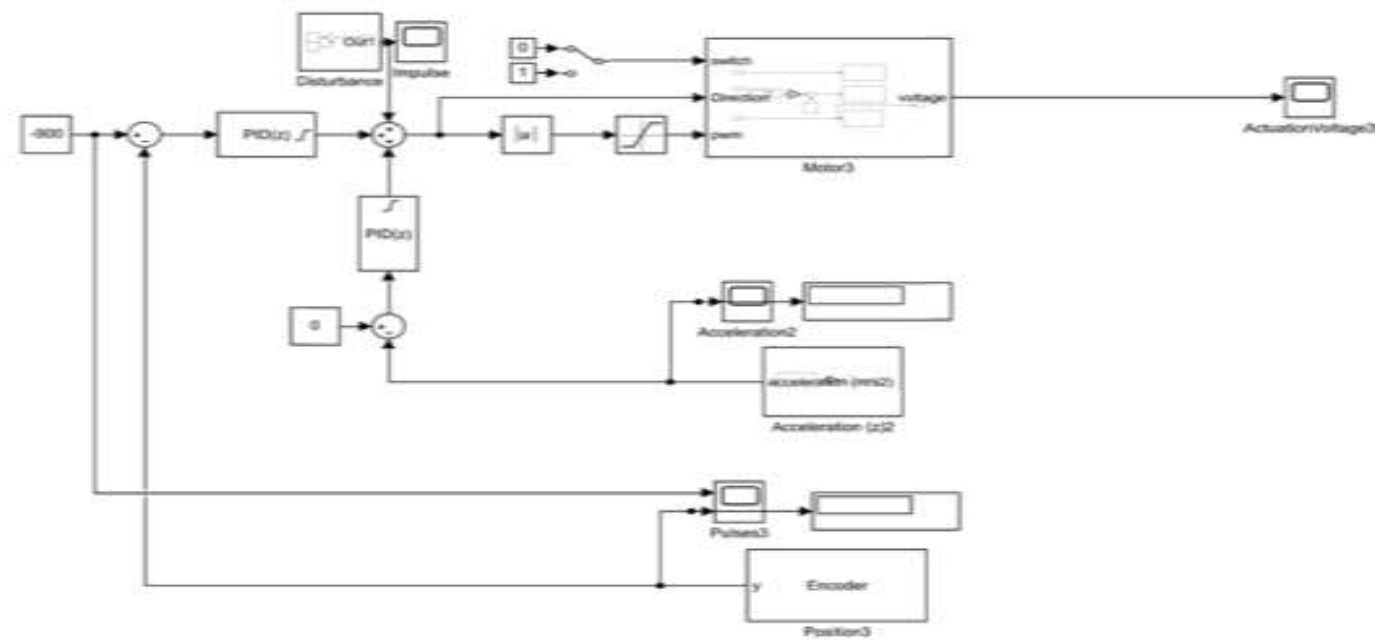

Figure 9. Simulink block diagram for parallel control with disturbance

\section{RESULTS}

The output data observed from the experiments were plotted through MATLAB figures which represent the trends for deflection of the beam and reference tracking for different cases.

\subsection{Position PD control with open loop deflection of beam response and different input waves}

Figure 10 shows the responses for the position control feedback. Figure 10 (a) illustrates the reference tracking achieved by the cart with different set-points. Figure 10 (b) shows a step response of the system where data points are marked. to compute the steady-state error (1\%), overshoot (1.2\%), and settling time $(0.3 \mathrm{~s})$. The steady-state error could be minimized by increasing the gain values, but it induces more vibration in the system and is a trade-off between the controller efficiency. Figure 10 (c) shows the control input for reference tracking generated by the PD gain.

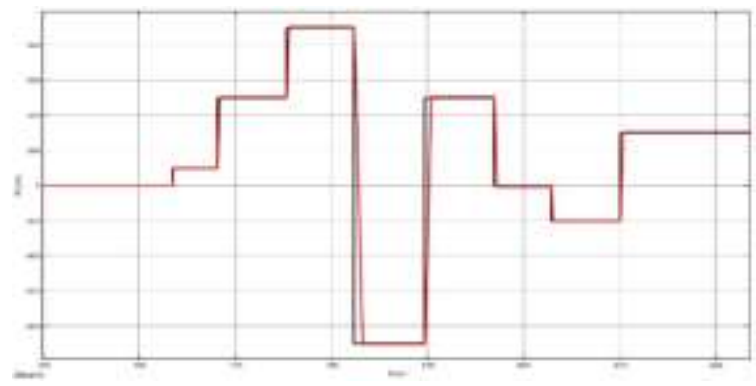

(a)

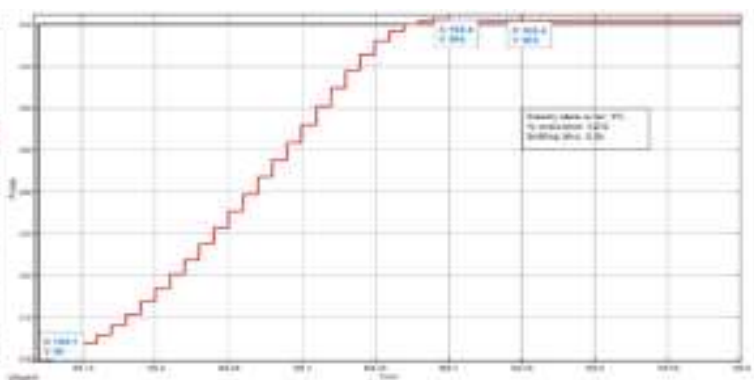

(b)

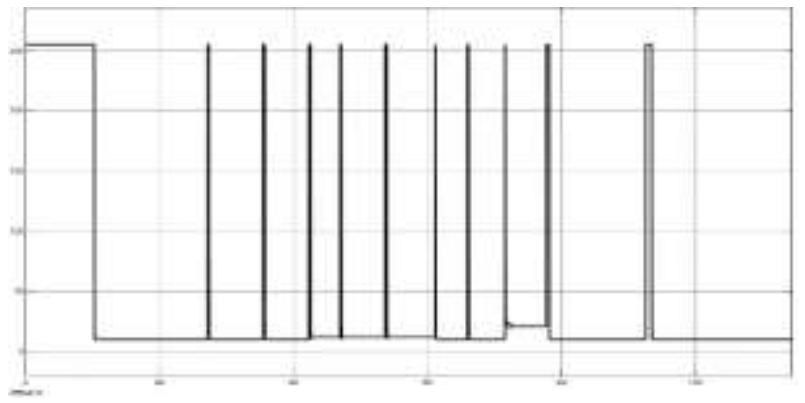

(c)

Figure 10. Position feedback control: (a) reference track, (b) step response, (c) control input for reference tracking 
Figure 11 shows the deflection response with the position control loop. Figure 11 (a) and 11 (b) shows the acceleration of beam in $\mathrm{x}, \mathrm{y}$, and $\mathrm{z}$ with and without the effect of gravity in the $\mathrm{y}$-axis. Figure 11 (c) shows the zoomed view of the acceleration of the beam along all three axes. It can be seen that the least vibration is observed in the axis towards the base as there is no motion along that axis. A limited vibration is observed along the axis towards the fixed end of the beam mostly in the positive direction (towards the fixed end). However, a significant vibration is observed along the axis of motion that oscillates in the positive and negative direction with the maximum oscillations. Therefore, with the mentioned observations, acceleration along the $\mathrm{z}$-axis was chosen to control the vibration of the beam and to calculate controller gain for the actuator input.

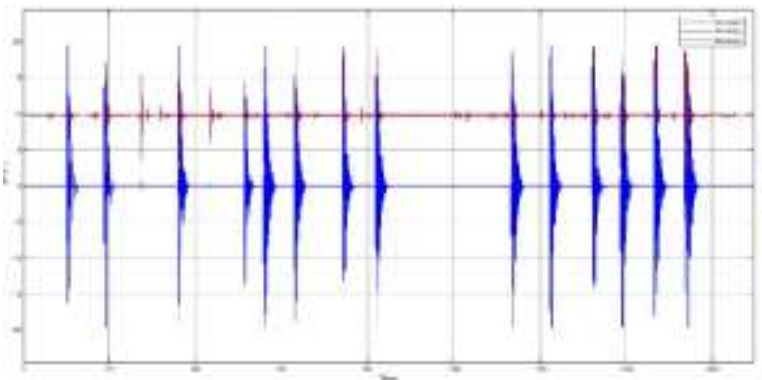

(a)

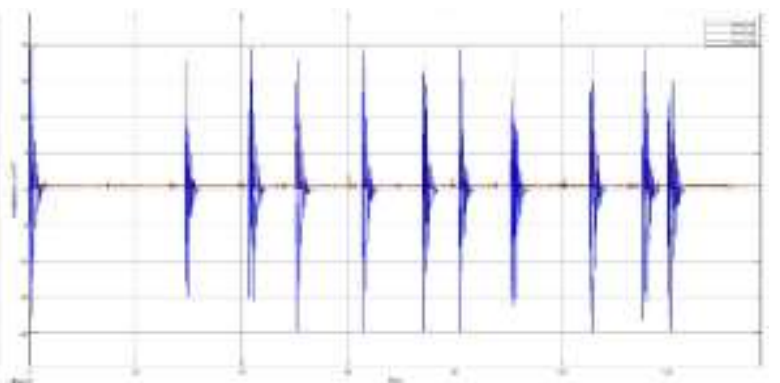

(b)

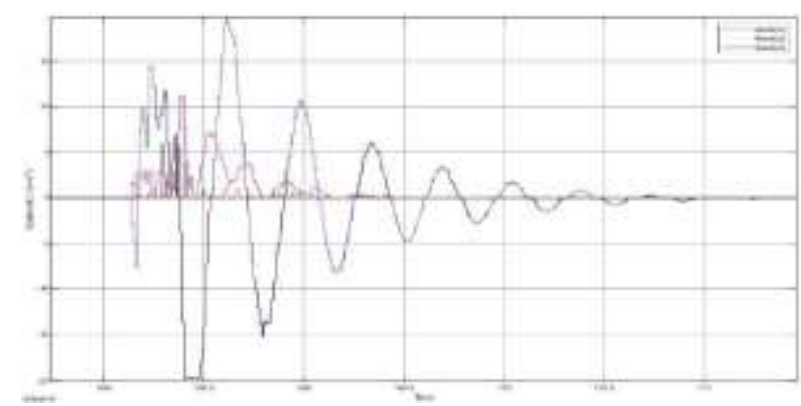

(c)

Figure 11. Open-loop beam deflection (x, y, z) response in terms of acceleration: (a) with the gravity effect

\subsubsection{Sine wave} and (b) without the gravity effect in terms of acceleration, and (c) the zoomed view

Figure 12 shows the system response when a sine wave is selected as the input signal for reference tracking. Figure 12 (a) shows the reference tracking for the sine wave of varying frequency and amplitude. Figure 12 (b) demonstrates the measured acceleration of the beam tip along the axis of motion, it can be seen that it is lesser with a lower frequency and increases with increasing frequency and amplitude. Figure 12 (c) shows the control input generated by the controller for the position control to track the sine wave, it can be concluded that the control input required is more for lesser frequency than the higher frequency for the same amplitude.

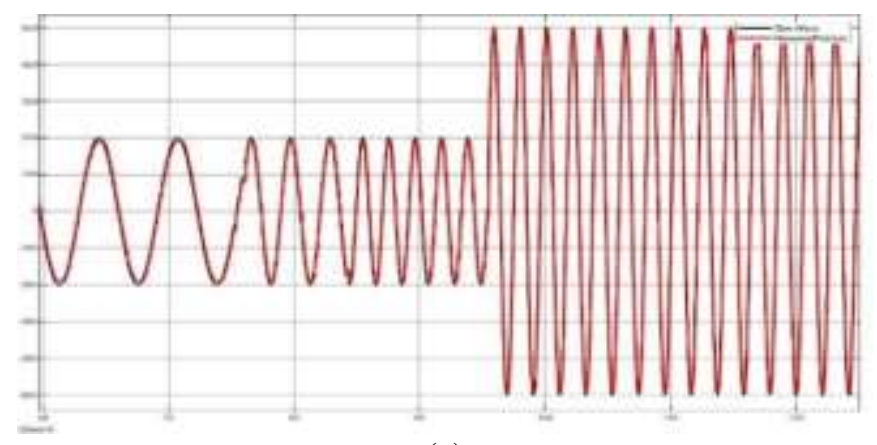

(a) 


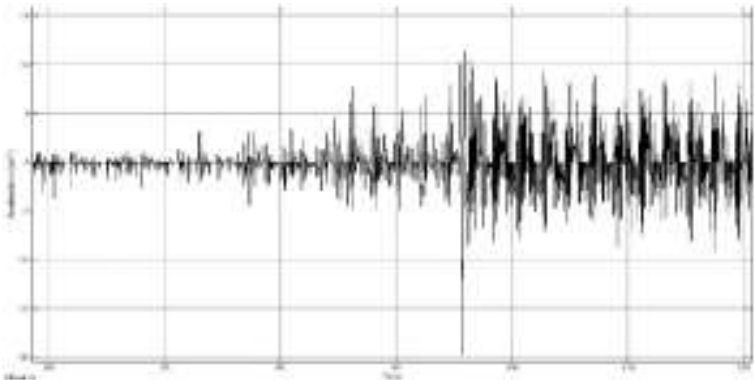

(b)

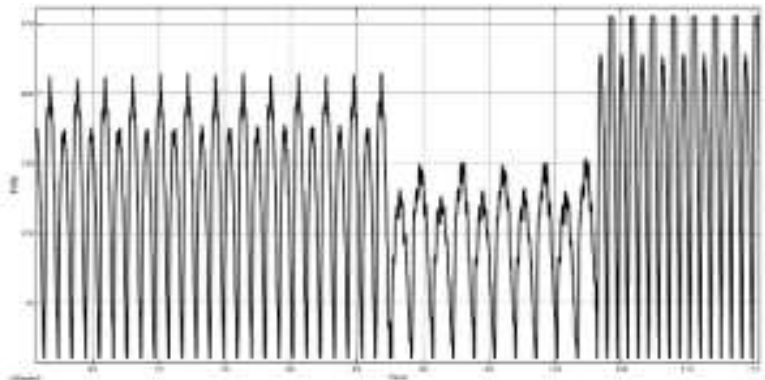

(c)

Figure 12. Sine wave (a) reference tracking and (b) sine wave deflection (z) response, and (c) the control input

\subsubsection{Square wave}

Figure 13 demonstrates the trend of reference tracking (a), deflection of beam open-loop response along the z-axis (b), and (c) the control input for the square wave. The trend observed is of higher amplitude than the one observed with a sine wave, this is because the motion is abrupt than the former.

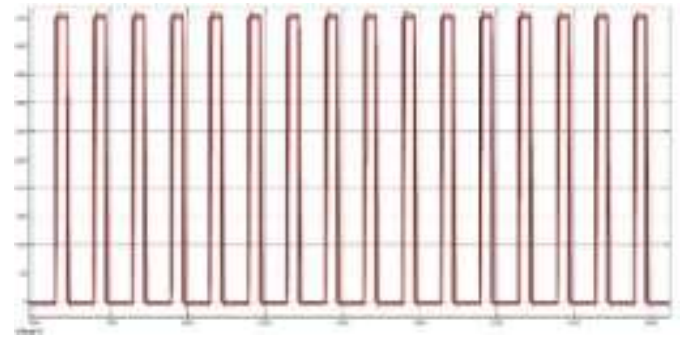

(a)

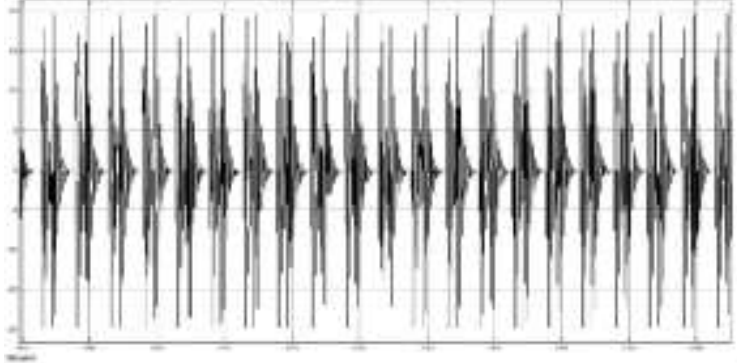

(b)

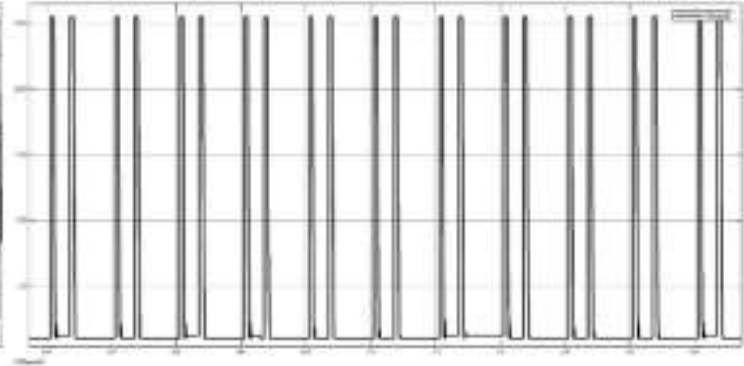

(c)

Figure 13. Square wave: (a) reference tracking and (b) square wave deflection(z) response, and (c) the control

\subsubsection{Triangle wave}

input

Figure 14 demonstrates (a) the trend of reference tracking, (b) the deflection at tip open-loop response along the z-axis, and (c) the control input for the triangle wave. The trend observed is of higher amplitude and with continuous variation than the one observed with the sine and square wave, this is because the motion inherits stationary points which induce more vibration.

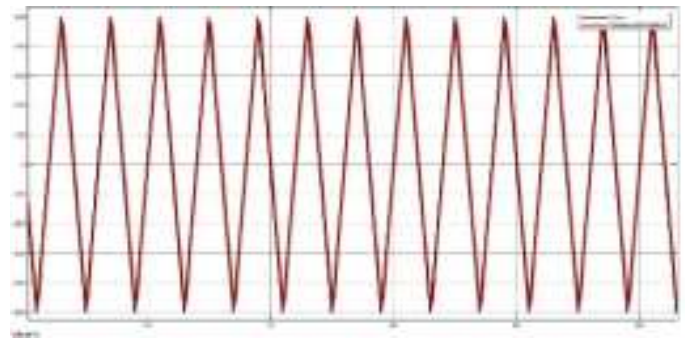

(a) 


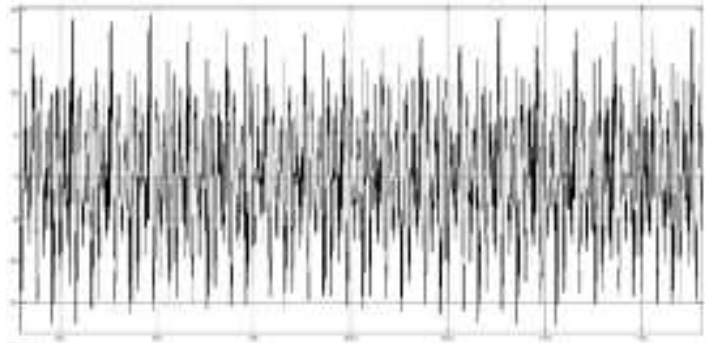

(b)

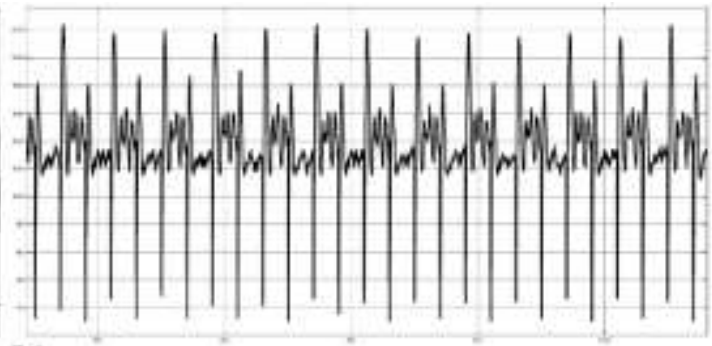

(c)

Figure 14. Triangle wave: (a) reference tracking and (b) deflection (z) response, and (c) the control input

\subsection{Vibration $P$ and PD control}

After observing the open-loop deflection responses from various input signals, the Ziegler Nichols tuning method was adopted to iterate for the best gain values. First P-gain was selected to check the response and then later the PD-gain. The difference in vibration/deflection trend is shown in Figure 15 (a) for openloop with $4 \mathrm{~s}$ of settling time, P closed-loop with $2 \mathrm{~s}$ of settling time, and PD closed-loop with $1.6 \mathrm{~s}$ of settling time. Figure 15 (b) shows the control input for P and PD controllers. It can be seen that the control input required for the PD controller is more than the P controller. Therefore, to consider the settling time and control input $\mathrm{P}$ controller was adopted to design an efficient controller.

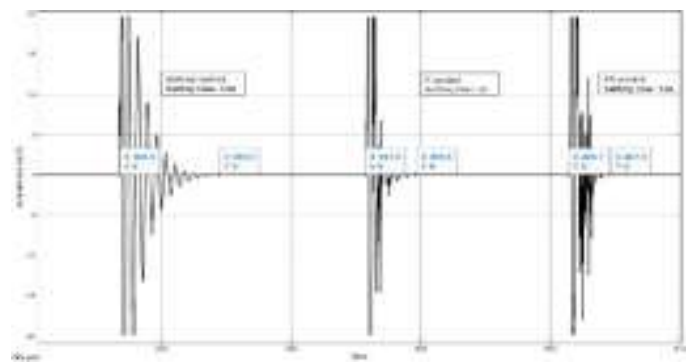

(a)

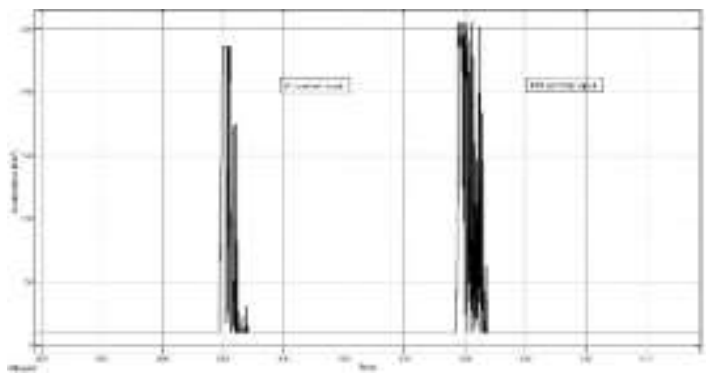

(b)

Figure 15. Vibration control through acceleration feedback in $\mathrm{z}$ (along with the motion): (a) acceleration (z) closed loop response, (b) control input

\subsection{Parallel Control}

Figure 16 shows the responses for position and vibration control loop using P and PD gains respectively, integrated using a parallel control loop. Figure 16 (a) demonstrates the reference tracking. Figure 16(b) illustrates the step response of the system with a steady-state error of $0.14 \%$, overshoot of $6.4 \%$, and the settling time of $1.7 \mathrm{~s}$. The overshoot is greater than the response with no acceleration feedback, however, the other parameters are lesser. The overshoot signifies the increased instability required to stabilize the system at the end of the motion. Figure 16 (c) shows the deflection at the tip of beam responses without and with feedback. Figure 16 (d) demonstrates the control input needed to stabilize the system; it can be seen that the control input for parallel loop requires constant input to minimize the vibration during the motion.

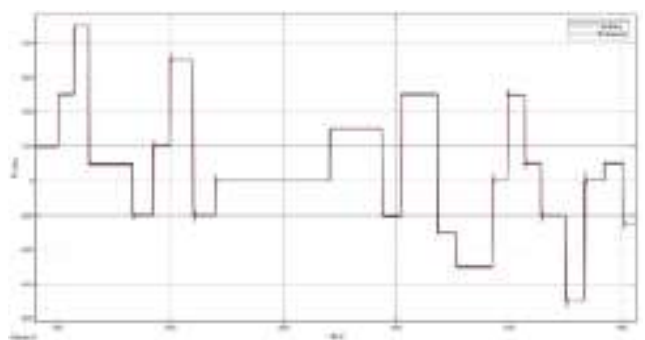

(a)

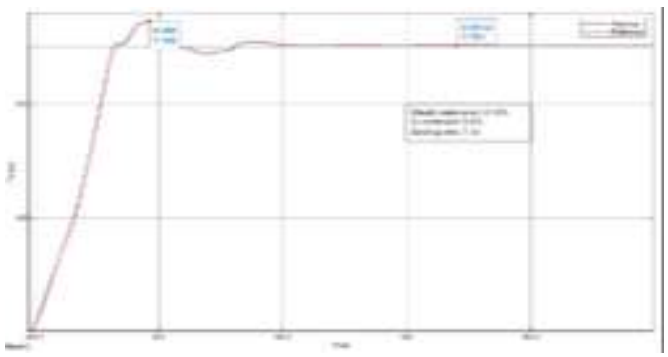

(b) 


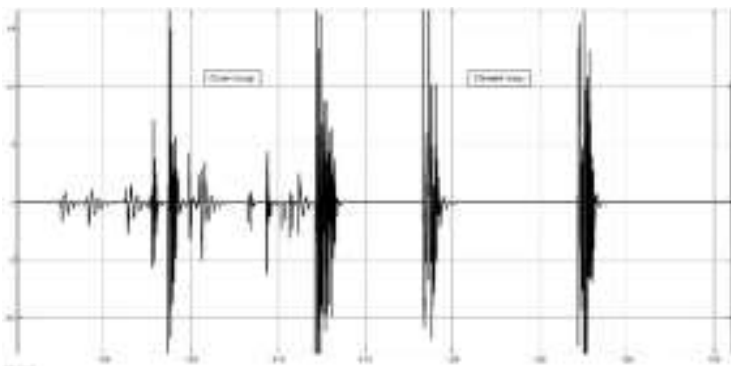

(c)

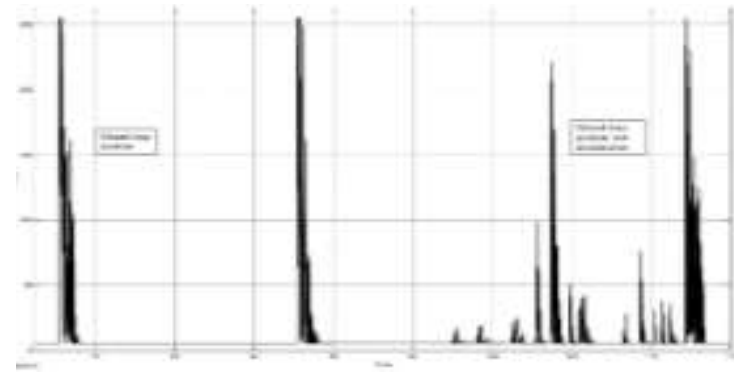

(d)

Figure 16. System response with parallel control for position and acceleration feedback: (a) reference tracking with and (b) step response for beam position and deflection control, (c) deflection (z) response, and (d) control input

\subsubsection{Disturbance rejection}

The results were obtained for the disturbance rejection applied by two methods. Figure 17 (a) shows set-point control after an impulse of amplitude 200 is applied to the system (directly to the cart which transfers the vibration to the beam) for $0.5 \mathrm{~s}$. The settling time to reach back to the set position was observed to be $1.7 \mathrm{~s}$ with a steady-state error of $0.11 \%$. Figure 17 (b) demonstrates the vibration control for the same impulse force with a settling time of $2.1 \mathrm{~s}$ for the vibration to be completely zero. Figure 17 (c) illustrates the disturbance rejection for position control when the external disturbance is applied directly to the beam inducing vibration. It can be observed that the settling time is $1.2 \mathrm{~s}$. Figure 17 (d) shows the disturbance rejection for vibration with the same external force which makes the settling time to be $1.8 \mathrm{~s}$.

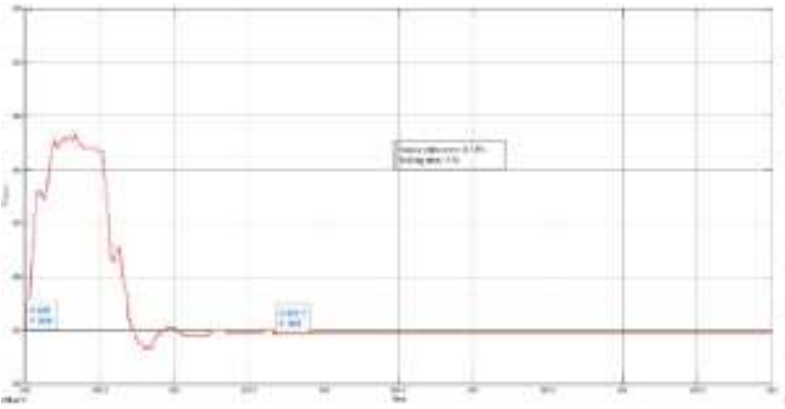

(a)

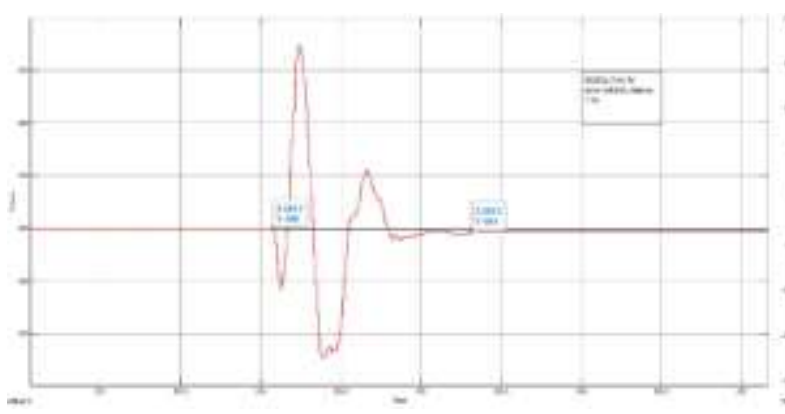

(c)

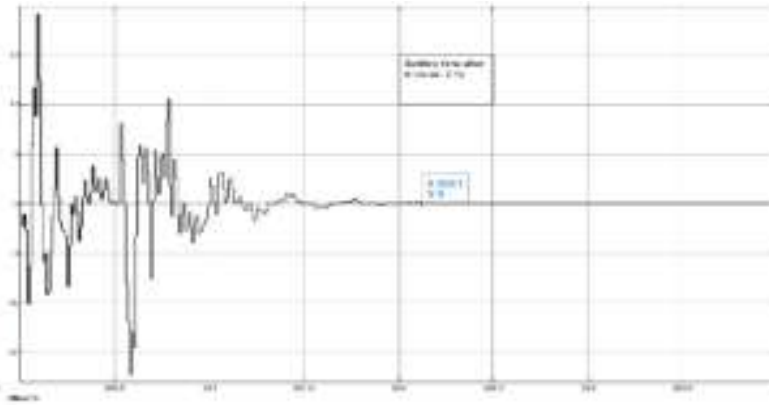

(b)

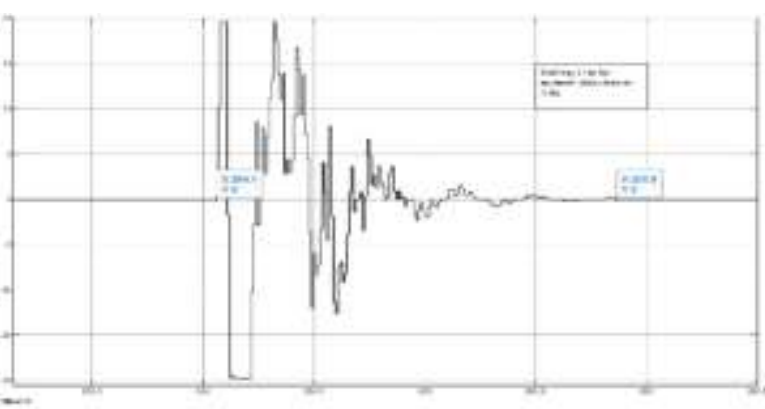

(d)

Figure 17. Parallel control with disturbance rejection: (a) position control and (b) beam deflection control for impulse disturbance, and (c) position control and (d) beam deflection control for external disturbance

\section{CONCLUSION AND FUTURE WORK}

A parallel P-PD controller scheme is discussed in this report to achieve the vibration and position control of a flexible beam. The open-loop trends of beam deflection along the axis of motion (z) are shown with the position feedback loop applying different input wave signals (sine, square, and triangle). Later, to

Parallel P-PD controller to achieve vibration and position control of a flexible beam (Ammar N. Abbas) 
control the vibration of the beam, an acceleration feedback loop is designed to stabilize the beam vibration/deflection of the beam. After observing the trend and tuning through the Ziegler Nichols method a parallel feedback control block diagram is modelled to achieve position reference tracking along with the vibration control. The real-time hardware implementation is achieved, and the responses were shown through plotted graphs of reference tracking, deflection (z), and control input to the actuator. In the future, other control strategies can be applied to minimize the error and achieve better stabilization and robustness. To minimize the noise and error in measurement, a deflection sensor (piezo or strain gauge) can be used at the fixed end of the beam by using sensor fusion.

\section{REFERENCES}

[1] W. He, J. Liu, Active Vibration Control and Stability Analysis of Flexible Beam Systems. Singapore: Springer Link, 2019, pp. 1-99.

[2] T. Zhang, K. Lin, and A. Zhang, "Research on flexible dynamics of a 6-DOF industrial robot and residual vibration control with a pre-adaptive input shaper," Journal of Mechanical Science and Technology, vol. 33, no. 4, pp. 18751889, 2019.

[3] P. M. Meshram and R. G. Kanojiya, "Tuning of PID controller using Ziegler-Nichols method for speed control of DC motor," in IEEE-International Conference on Advances in Engineering, Science and Management (ICAESM 2012), 2012, pp. 117-122.

[4] M. Dadfarnia, N. Jalili, Z. Liu, and D. M. Dawson, "An observer-based piezoelectric control of flexible Cartesian robot arms: theory and experiment," Control Engineering Practice, vol. 12, no. 8, pp. 1041-1053, 2004.

[5] J. Hu, D. Zhu, Q. Chen, and Q. Gu, "Active Vibration Control of a Flexible Beam Based on Experimental Modal Analysis," 2011 3rd International Conference on Advanced Computer Control ICACC. 2011.

[6] Z. Qiu, J. Han, X. Zhang, Y. Wang, and Z. Wu, "Active vibration control of a flexible beam using a non-collocated acceleration sensor and piezoelectric patch actuator," Journal of Sound and Vibration, vol. 326, no. 3-5, pp. 438455, 2009.

[7] G. Song and H. Gu, "Active vibration suppression of a smart flexible beam using a sliding mode-based controller," Journal of Sound and Vibration, vol 13, no. 8, pp. 1095-1107, 2007.

[8] B. Guo and C. Xu, "The Stabilization of a One-Dimensional Wave Equation by Boundary Feedback with Noncollocated Observation," IEEE Transactions on Automatic Control, vol. 52, no. 2, pp. 371-377, 2007.

[9] A. I. Ozdagli, B. Liu, and F. Moreau, "Low-cost, efficient wireless intelligent sensors (LEWIS) measuring realtime reference-free dynamic displacements," Mechanical Systems and Signal Processing, vol. 107, pp. 343-356, 2018.

[10] Z. Qiu, J. Han, X. Zhang, Y. Wang, and Z. Wu, "Active vibration control of a flexible beam using a non-collocated acceleration sensor and piezoelectric patch actuator," Journal of sound and vibration, vol. 326, no. 3-5, pp. 438455, 2009.

[11] Y. Orlov, L. Perez, O. Gomez, and L. Autrique, "ISS output feedback synthesis of disturbed reaction-diffusion processes using non-collocated sampled-in-space sensing and actuation," Automatica, vol. 122, p. 109257, 2020.

[12] K. Yamaguchi, T. Endo, Y. Kawai, and F. Matsuno, "Non-collocated boundary control for contact-force control of a one-link flexible arm," Journal of the Franklin Institute, vol. 357, no. 7, pp. 4109-4131, 2020.

[13] A. Bazaei, M. Boudaoud, M. H. Ettefagh, Z. Chen, and S. Régnier, "Non-Collocated Displacement Sensing by Semiconductor Strain Gauges in Differentially Piezo-Driven Nanopositioners," IEEE Sensors Journal, vol. 21, no. 8, pp. 9690-9697, 2021.

[14] B. Z. Guo and T. Meng. "Robust error based non-collocated output tracking control for a heat equation." Automatica, 114, 2020: 108818 .

[15] M. Morlock, N. Meyer, M.-A. Pick, and R. Seifried, "Real-time trajectory tracking control of a parallel robot with flexible links," Mechanism and Machine Theory, vol. 158, p. 104220, 2021. 